\author{
Mirosław SIERGIEJCZYK ${ }^{1}$, Adam ROSIŃSKI ${ }^{1}$, Jacek PAŚ ${ }^{2}$ \\ ${ }^{1}$ Warsaw University of Technology (Politechnika Warszawska) \\ 2 Military University of Technology (Wojskowa Akademia Techniczna)
}

\title{
ELECTROMAGNETIC INTERFERENCE ISSUE IN SAFETY SYSTEMS APPLIED AT AIRPORTS
}

\begin{abstract}
The proper operation of electronic devices used in transport facilities is possible by protecting them against the effect of adverse electromagnetic fields. At present, all kinds of facilities, including critical infrastructure facilities, intended for functioning in peace conditions and emergency states, are equipped with power systems and numerous electronic devices, including electronic safety systems. On the basis of observing the process of their operation, it can be concluded that an artificially generated intended or unintended electromagnetic field, within various frequency ranges, may negatively affect the operation of electronic devices. It was found that the operation of the electronic safety systems applied at airports may be subject to interference as a result of the impact of adverse electromagnetic fields. Therefore, it is necessary to analyse the impact of electromagnetic interference on the operation of these systems. This article presents a developed author's operation process model including electromagnetic interference within low frequencies. The reliability and operation analysis of the electronic safety systems carried out by the authors allows for numerical assessment of various types of solutions both technical and organisational ones. As a result, they can be implemented, in order to minimise the impact of electromagnetic interference on the system functioning. This is a new approach to this issue, as there are currently designed and installed security systems at airports, but there is no analysis of the impact of electromagnetic interference on their functioning. In further research concerning this issue, the authors plan to further develop reliability-operational models, which will differentiate between the states of partial fitness. It will allow for more accurate mapping of the operation of the electronic safety systems which are applicable at airports.
\end{abstract}

Keywords: operation, electromagnetic interference, safety systems, modelling 


\section{Introduction}

The proper operation of electronic devices is possible by protecting them against the effect of adverse electromagnetic fields [2,14]. At present, all kinds of structures (also at airports) are equipped with electrical systems and numerous electronic devices, including electronic safety systems [6, 7, 19, 20]. Different types of telecommunication systems are necessary to provide certain services to passengers and carriers within intelligent transport systems [5, 10, 12]. On the basis of numerous observations, it is possible to conclude that artificially generated electric and magnetic fields of different frequency ranges may adversely affect the operation of electronic devices [18]. It was found that the operation of electronic devices may be seriously interfered due to the effect of adverse electromagnetic fields. Therefore, the issues related to the analysis of electromagnetic fields, which are generated by the electronic safety system components, are so important. The article presents the results of measuring the electromagnetic fields of detectors applied in the safety systems. The developed author's operation process model including electromagnetic interference within low frequencies was also demonstrated.

The increase in the safety level of electronic alarm systems applied at airports can be obtained, among others, by improving their reliability parameters [12]. The entire system reliability affects both the reliability of components and the use of redundant structures. The first solution is aimed at preventing damage. In the second case, the use of redundancy, although leading to the device expansion, enables the occurring damage tolerance (through the introduction of partial fitness states). The redundancy may apply both to the device components and to external power systems (which is particularly important for facilities classified as the critical infrastructure). The quality of information [21, 22] obtained by the devices from detectors $[3,16]$ is also important during the reliability-operational analysis. In some research papers, it is suggested to use artificial neural networks [4] or fuzzy logic. Oscillations [1] also significantly affect the operation of the electronic safety systems, although they are not included in this paper.

In order to reduce the electromagnetic interference effects on the operation of the electronic safety systems, various design solutions are applied. The publication [11] presents the use of various materials for the construction of mobile means of transport and their impact (including screening) on satisfying the electromagnetic compatibility requirements.

A separate group of publications include deliberations in the field of electromagnetic interference and electromagnetic compatibility of the safety systems $[23,24]$. A fairly large group of publications refers to the CCTV systems 
$[8,9]$. In these developments, the authors approach this subject in a general manner, without taking into account the specificity of airports.

\section{Safety systems}

The risk alarm system is a set of technical measures and tactical rules aimed at providing a certain object (human and/or property) with an appropriate safety level within a strictly specified space and time. The electronic protection system is created most often with the use of the following systems distinguished depending on detected threats, as the following systems:

- intrusion and hold-up systems (SSWiN),

- fire alarm systems (SSP),

- access control systems (SKD),

- CCTV systems,

- external site protection systems.

The protection resulting from operation of these systems can be supplemented by the following systems:

- health condition and personal threat alarm systems,

- environmental threat alarm systems,

- anti-theft systems,

- voice alarm systems,

- car protection systems against burglary and theft.

One of the most important alarm system elements includes alarm transmission systems. They constitute devices or networks used for transferring information on the condition of one or more alarm systems to one or more alarm receiving centres (ACO) [14]. Currently, in addition to a local signalling device in the protected facility of the threat state, it is strived for sending information about the threat to the alarm receiving centre.

The Intrusion and Hold-up System is designed to detect and signal the state of danger to property and/or people. The European standard EN 50131-1:2006 "Alarm systems - Intrusion and hold-up systems - Part 1: System requirements", which at the same time, has the status of the Polish Standard PN-EN 50131-1:2009 "Alarm systems - Intrusion and hold-up systems - System requirements", contains a list of components (elements), which should be included in the Intrusion and Hold-up System [17]:

- control panel,

- one or more sensors, 
- one or more signalling devices and/or alarm transmission systems,

- basic power supply,

- backup power supply.

The connections between the Intrusion and Hold-up System elements should meet specific requirements included in the standards, and at the same time, they must also correspond to the manufacturer's acceptable parameters. Therefore, all types of systems - that meet the requirements included in the PN-EN 50131-1:2009 standard on the safety level - are manufactured. The following grades are distinguished:

- grade 1: Low risk (it is assumed that the intruder has minimum knowledge on the alarm system and $\mathrm{s} / \mathrm{he}$ has readily available tools with a limited choice),

- grade 2: Low to medium risk (it is assumed that the intruder has minimum knowledge on the alarm system and s/he has widely available tools and portable devices, e.g. multimeter),

- grade 3: Medium to high risk (it is assumed that the intruder is fluent in the alarm system and $\mathrm{s} / \mathrm{he}$ has a complex set of advanced tools and portable electronic equipment),

- grade 4: High risk (it is applied when safety takes priority over all other factors. It is assumed that the intruder has the abilities or measures to plan a burglary in detail, and s/he has a set of any equipment, including measures to replace key elements of the electronic alarm system.

After determining the protection degree to be applied in the protected facility, the intrusion and hold-up system, the devices of which meet the requirements within at least one adopted level, is designed. At airports, it is also important to take into account the impact of electromagnetic radiation, which is emitted by individual elements of the Intrusion and Hold-up System, and radiation that affects these elements from other devices installed at the airport. The authors presented such an approach in this article.

\section{The study of the electromagnetic field generated by the safety system detectors}

Electronic devices used in air transport are operated within the specified electromagnetic environment. The electromagnetic environment shaped by the natural phenomena that occur on the ground is seriously distorted in the industrialised areas. It results from the occurrence of a large number of electromagnetic field 
sources radiating in an intended or unintended way. Every electrically powered electronic or electrical device generates its own electromagnetic field, which is associated with its operation. Within the airport area, there are electronic devices, which should operate properly regardless of the interference that have an impact on them. The mutual coexistence of the systems as well as their proper functioning within the airport area in the particular electromagnetic environment and operation without the implementation of unacceptable distortion to the environment can be defined as electromagnetic compatibility.

The requirements of the modern electronic devices applied, among others, in air transport, include, inter alia, miniaturisation, limited energy consumption and high reliability. The implementation of these restrictions results in the fact that a level of signals of the useful devices can be compared to the level of interference generated e.g. by static and mobile interference sources (e.g. radiolocation, base and radio stations, TV, medium- and high-voltage lines, transformer stations, and commonly used electrical devices).

In case of the impact of electromagnetic interference on electronic transport systems, it is possible to distinguish four operating states:

- the system does not react to external and internal interference - the interference level is low and the permissible interference levels were not exceeded, the system remains in a given operating state, in which it currently is,

- the devices making up a control system automatically remove interference with the use of the applied passive or active filters, screening, adequate arrangement or systemic solutions,

- occurrence of interference with values not exceeding the permissible ones causes the system transition from the state of fitness to the state of partial fitness - restoring the state of fitness requires taking specific activities,

- occurrence of interference with values exceeding the permissible ones damages the system, and therefore, it results in the system transition from the state of fitness to the state of unfitness.

The parameters of the unintended electromagnetic field generated by individual elements of the electronic safety system constitute a function of numerous variables - i.e., among others, power supply (DC or AC), the current consumed by a given device in various types of operation, e.g. alarm, diagnosis and standby states, etc.

One of the most important elements of the intrusion and hold-up systems includes detection elements - detectors. They indicate the protected zone violation. They are characterised by a different emission level of the unintended magnetic field - fig. 1 ("Bx" symbol was used for marking the maximum value for components in 
three planes $\mathrm{x}, \mathrm{y}, \mathrm{z}$ ), and the electric field - fig. 2. The largest B induction value of the magnetic field within the ELF frequency range was obtained for a dual detector that uses the electromagnetic radiation analysis within the infrared and microwave range (PIR + MW type detector) in order to generate the alarm state.

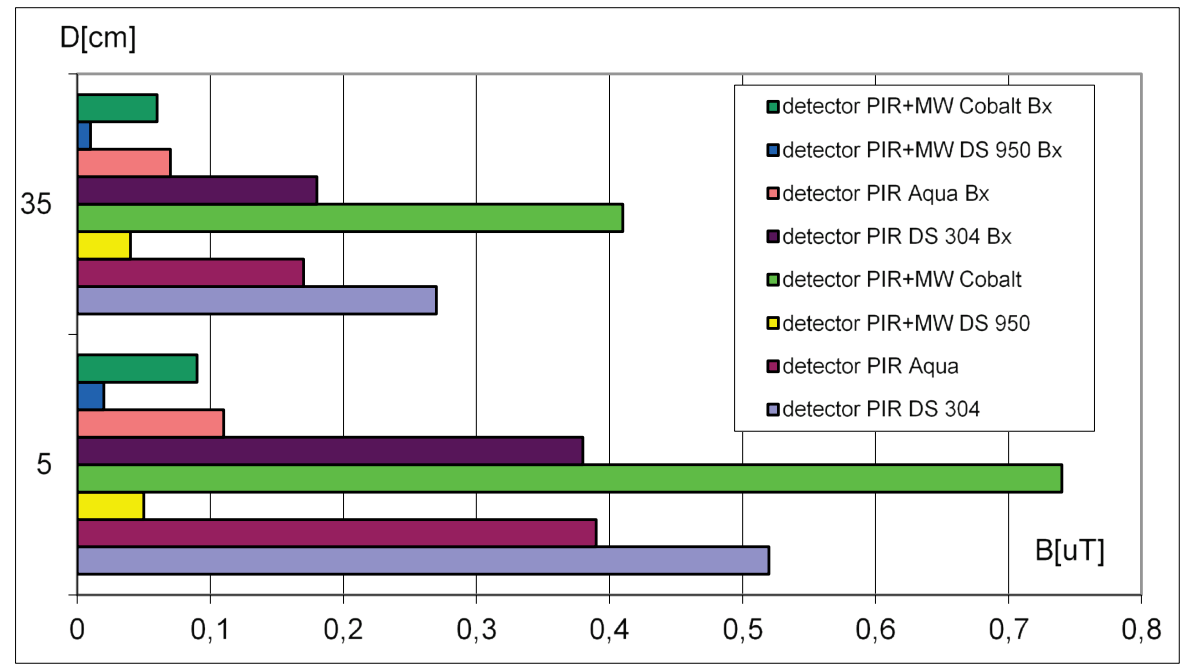

Fig. 1. The B induction measurement results of the magnetic field within the ELF frequency range at a distance of 5 and $35 \mathrm{~cm}$ from the casing for various types of alarm detectors used in the alarm system

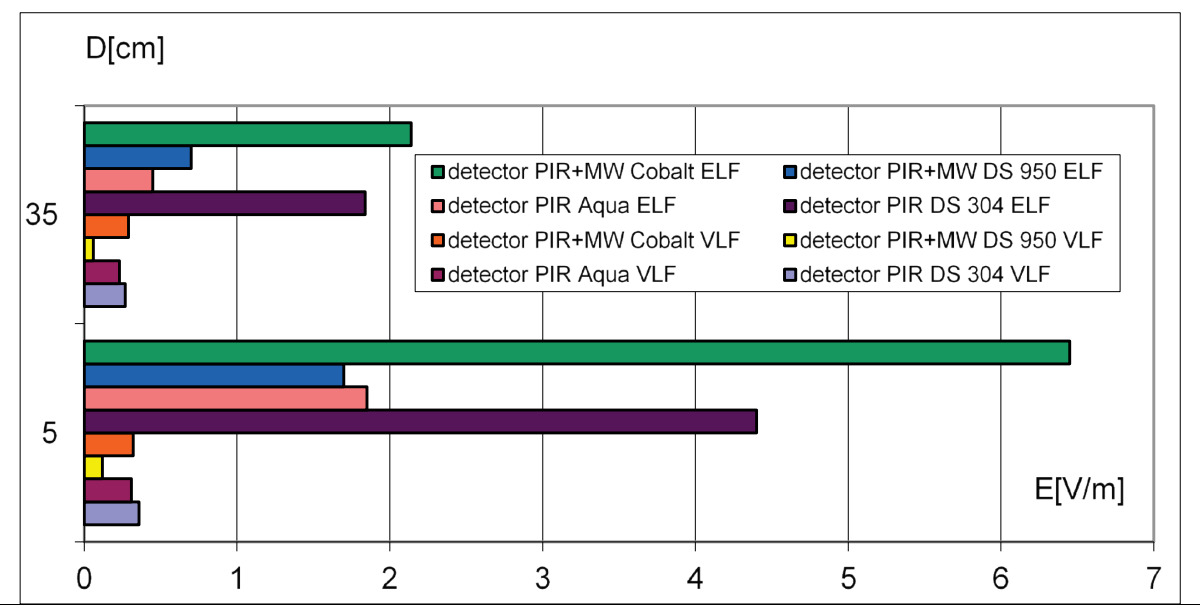

Fig. 2. The E intensity measurement results of the electric field within the ELF and VLF frequency ranges at a distance of 5 and $35 \mathrm{~cm}$ from the casing for various types of alarm detectors used in the alarm system 
As a result of the carried-out measurements, it was found that the greatest intensity of the electric and magnetic fields occurs in active detectors. An example of it includes PIR + MW dual detectors, in which, inter alias, the Doppler effect is applied. Therefore, the reliability-operational analysis should be carried out in order to determine the electromagnetic radiation impact on the electronic safety system operation.

\section{Reliability and operation modelling of the electronic safety system including electromagnetic interference}

The operation process modelling of the electronic safety systems used at airports, including electromagnetic interference, require the analysis of their functioning in actual conditions (also including the diagnostic signals). Such an attitude makes it possible to obtain the relationships that allow to determine the values of probabilities of the considered systems staying in the distinguished operating states.

By conducting the operation analysis of the electronic safety systems used at airports, including electromagnetic interference, it is possible to illustrate the relationships occurring in this structure, in terms of reliability and operation, as it is presented in fig. 3. Markings in fig. 3:

$\mathrm{R}_{\mathrm{O}}(\mathrm{t})$ - probability function of the system staying in the state of full fitness Spz, $\mathrm{Q}_{\mathrm{ZB1}}(\mathrm{t})$ - probability function of the system staying in the state of impendency over safety $\mathrm{S}_{\mathrm{ZB} 1}$,

$\mathrm{Q}_{\mathrm{ZB} 2}(\mathrm{t})$ - probability function of the system staying in the state of impendency over safety $\mathrm{S}_{\mathrm{ZB} 2}$,

$\mathrm{Q}_{\mathrm{B}}(\mathrm{t})$ - probability function of the system staying in the state of safety unreliability $\mathrm{S}_{\mathrm{B}}$,

$\lambda_{Z B 1}-$ intensity of transitions from the state of full fitness $S_{P Z}$ to the state of impendency over safety $S_{\mathrm{ZB} 1}$,

$\lambda_{\mathrm{ZB} 2}-$ intensity of transitions from the state of impendency over safety $\mathrm{S}_{\mathrm{ZB} 1}$ to the state of impendency over safety $\mathrm{S}_{\mathrm{ZB} 2}$,

$\lambda_{\mathrm{ZB} 3}-$ intensity of transitions from the state of impendency over safety $\mathrm{S}_{\mathrm{ZB} 2}$ to the state of safety unreliability $\mathrm{S}_{\mathrm{B}}$,

$\mu_{\mathrm{ZB} 1} \quad-$ intensity of transitions from the state of impendency over safety $\mathrm{S}_{\mathrm{ZB} 1}$ to the state of full fitness $\mathrm{S}_{\mathrm{PZ}}$,

$\mu_{\mathrm{ZB} 2}-$ intensity of transitions from the state of impendency over safety $\mathrm{S}_{\mathrm{ZB} 2}$ to the state of impendency over safety $\mathrm{S}_{\mathrm{ZB} 1}$, 
$\mu_{\mathrm{ZB} 3}-$ intensity of transitions from the state of safety unreliability $\mathrm{S}_{\mathrm{B}}$ to the state of impendency over safety $\mathrm{S}_{\mathrm{ZB} 2}$,

$\mu_{\mathrm{B} 0} \quad-$ intensity of transitions from the state of impendency over safety $\mathrm{S}_{\mathrm{ZB} 2}$ to the state of full fitness $\mathrm{S}_{\mathrm{PZ}}$,

$\mu_{\mathrm{B} 1} \quad-$ intensity of transitions from the state of full fitness $\mathrm{S}_{\mathrm{PZ}}$ to the state of safety unreliability $\mathrm{S}_{\mathrm{B}}$.

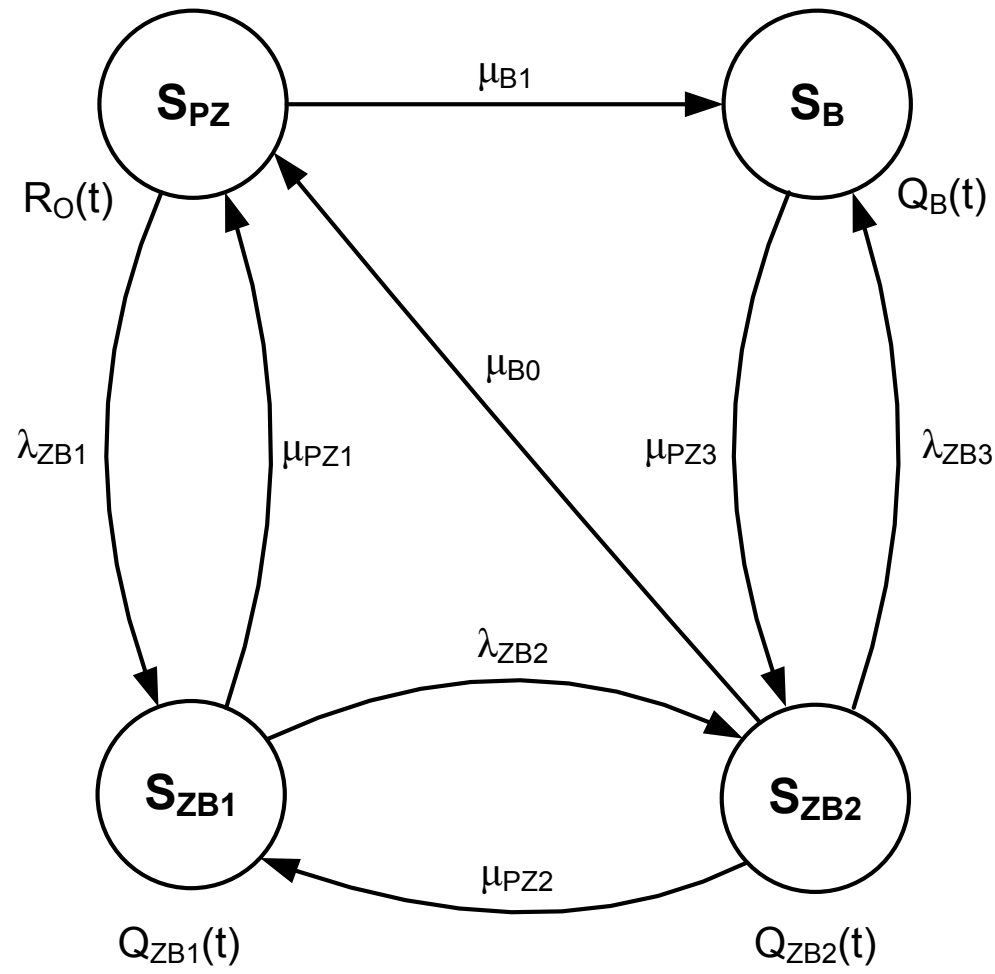

Fig. 3. Relationships in the electronic safety system including electromagnetic interference

If the electronic safety system is in the state of full fitness $\mathrm{S}_{\mathrm{PZ}}$ and there is electromagnetic interference (in the acceptable range), the system transits to the state of impendency over safety $\mathrm{S}_{\mathrm{ZB} 1}$ with the intensity $\lambda_{\mathrm{ZB} 1}$. If the system is in the state of impendency over safety $\mathrm{S}_{\mathrm{ZB} 1}$, it is possible to transit into the state of full fitness $S_{P Z}$ with the intensity $\mu_{\mathrm{ZB} 1}$ provided that the activities on reducing the electromagnetic interference consequences are undertaken.

If the electronic safety system is in the state of impendency over safety $\mathrm{S}_{\mathrm{ZB} 1}$ and there is electromagnetic interference (exceeding the permissible values, but the system still operates), the system transits to the state of impendency over safety 
$\mathrm{S}_{\mathrm{ZB} 2}$ with the intensity $\lambda_{\mathrm{ZB} 2}$. If the system is in the state of impendency over safety $\mathrm{S}_{\mathrm{ZB} 2}$, it is possible to transit into the state of impendency over safety $\mathrm{S}_{\mathrm{ZB} 1}$ with the intensity $\mu_{\mathrm{ZB} 2}$ provided that the activities on reducing the electromagnetic interference consequences (to the level of permissible values) are undertaken.

If the electronic safety system is in the state of impendency over safety $\mathrm{S}_{\mathrm{ZB} 2}$ and there is electromagnetic interference (exceeding the permissible values and resulting in the system operation interruption), the system transits to the state of safety unreliability $S_{B}$ with the intensity $\lambda_{Z B 3}$. If the system is in the state of safety unreliability $S_{B}$, it is possible to transit into the state of impendency over safety $S_{Z B 2}$ with the intensity $\mu_{\mathrm{ZB} 3}$ provided that the activities on reducing the electromagnetic interference consequences (to the level exceeding the permissible values, but the system still operates) are undertaken.

If the electronic safety system is in the state of impendency over safety $\mathrm{S}_{\mathrm{ZB} 2}$, it is possible to transit into the state of full fitness $\mathrm{S}_{\mathrm{PZ}}$ with the intensity $\mu_{\mathrm{B} 0}$ provided that the activities on reducing the electromagnetic interference consequences (to the level below the permissible values) are undertaken.

If the electronic safety system is in the state of full fitness $\mathrm{S}_{\mathrm{PZ}}$, it is possible to transit into the state of safety unreliability $S_{B}$ with the intensity $\mu_{\mathrm{B} 1}$ in case of the occurrence of the electromagnetic interference exceeding the permissible values and resulting in the system operation interruption.

The following Chapman-Kolmogorov equations can be used to describe the system presented in fig. 3:

$$
\begin{gathered}
R_{0}^{\prime}(t)=-\lambda_{Z B 1} \cdot R_{0}(t)+\mu_{P Z 1} \cdot Q_{Z B 1}(t)+\mu_{B 0} \cdot Q_{Z B 2}(t)-\mu_{B 1} \cdot R_{0}(t) \\
Q_{Z B 1}^{\prime}(t)=\lambda_{Z B 1} \cdot R_{0}(t)-\mu_{P Z 1} \cdot Q_{Z B 1}(t)-\lambda_{Z B 2} \cdot Q_{Z B 1}(t)+\mu_{P Z 2} \cdot Q_{Z B 2}(t) \\
Q_{Z B 2}^{\prime}(t)=\lambda_{Z B 2} \cdot Q_{Z B 1}(t)-\mu_{P Z 2} \cdot Q_{Z B 2}(t)-\lambda_{Z B 3} \cdot Q_{Z B 2}(t)+\mu_{P Z 3} \cdot Q_{B}(t)-\mu_{B 0} \cdot Q_{Z B 2}(t) \\
Q_{B}^{\prime}(t)=\lambda_{Z B 3} \cdot Q_{Z B 2}(t)-\mu_{P Z 3} \cdot Q_{B}(t)+\mu_{B 1} \cdot R_{0}(t)
\end{gathered}
$$

If the baseline conditions are assumed:

$$
\begin{gathered}
R_{0}(0)=1 \\
Q_{Z B 1}(0)=Q_{Z B 2}(0)=Q_{B}(0)=0
\end{gathered}
$$

and the Laplace transform will be used, the following set of linear equations can be obtained: 


$$
\begin{gathered}
s \cdot R_{0}^{*}(s)-1=-\lambda_{Z B 1} \cdot R_{0}^{*}(s)+\mu_{P Z 1} \cdot Q_{Z B 1}^{*}(s)+\mu_{B 0} \cdot Q_{Z B 2}^{*}(s)-\mu_{B 1} \cdot R_{0}^{*}(s) \\
s \cdot Q_{Z B 1}^{*}(s)=\lambda_{Z B 1} \cdot R_{0}^{*}(s)-\mu_{P Z 1} \cdot Q_{Z B 1}^{*}(s)-\lambda_{Z B 2} \cdot Q_{Z B 1}^{*}(s)+\mu_{P Z 2} \cdot Q_{Z B 2}^{*}(s) \\
s \cdot Q_{Z B 2}^{*}(s)=\lambda_{Z B 2} \cdot Q_{Z B 1}^{*}(s)-\mu_{P Z 2} \cdot Q_{Z B 2}^{*}(s)-\lambda_{Z B 3} \cdot Q_{Z B 2}^{*}(s)+\mu_{P Z 3} \cdot Q_{B}^{*}(s)-\mu_{B 0} \cdot Q_{Z B 2}^{*}(s) \\
s \cdot Q_{B}^{*}(s)=\lambda_{Z B 3} \cdot Q_{Z B 2}^{*}(s)-\mu_{P Z 3} \cdot Q_{B}^{*}(s)+\mu_{B 1} \cdot R_{0}^{*}(s)
\end{gathered}
$$

Taking the above Laplace transform into account, the probabilities of the system staying in the considered functional states will take the form as below:

$$
\begin{aligned}
& R_{0}^{*}(s)=\frac{b \cdot \lambda_{Z B 3} \cdot \mu_{P Z 3}-b \cdot c \cdot d \cdot s+d \cdot s \cdot \lambda_{Z B 2} \cdot \mu_{P Z 2}}{a \cdot b \cdot \lambda_{Z B 3} \cdot \mu_{P Z 3}+b \cdot \mu_{B 0} \cdot \mu_{B 1} \cdot \mu_{P Z 3}+\mu_{B 1} \cdot \mu_{P Z 1} \cdot \mu_{P Z 2} \cdot \mu_{P Z 3}-} \\
& -\lambda_{Z B 1} \cdot \mu_{P Z 1} \cdot \lambda_{Z B 3} \cdot \mu_{P Z 3}+a \cdot d \cdot s \cdot \lambda_{Z B 2} \cdot \mu_{P Z 2}+ \\
& +c \cdot d \cdot s \cdot \lambda_{Z B 1} \cdot \mu_{P Z 1}+d \cdot s \cdot \mu_{B 0} \cdot \lambda_{Z B 1} \cdot \lambda_{Z B 2}-a \cdot b \cdot c \cdot d \cdot s \\
& Q_{Z B 1}^{*}(s)=-\frac{\mu_{B 1} \cdot \mu_{P Z 2} \cdot \mu_{P Z 3}-\lambda_{Z B 1} \cdot \lambda_{Z B 3} \cdot \mu_{P Z 3}+c \cdot d \cdot s \cdot \lambda_{Z B 1}}{a \cdot b \cdot \lambda_{Z B 3} \cdot \mu_{P Z 3}+b \cdot \mu_{B 0} \cdot \mu_{B 1} \cdot \mu_{P Z 3}+\mu_{B 1} \cdot \mu_{P Z 1} \cdot \mu_{P Z 2} \cdot \mu_{P Z 3}-} \\
& -\lambda_{Z B 1} \cdot \mu_{P Z 1} \cdot \lambda_{Z B 3} \cdot \mu_{P Z 3}+a \cdot d \cdot s \cdot \lambda_{Z B 2} \cdot \mu_{P Z 2}+ \\
& +c \cdot d \cdot s \cdot \lambda_{Z B 1} \cdot \mu_{P Z 1}+d \cdot s \cdot \mu_{B 0} \cdot \lambda_{Z B 1} \cdot \lambda_{Z B 2}-a \cdot b \cdot c \cdot d \cdot s \\
& Q_{Z B 2}^{*}(s)=-\frac{b \cdot \mu_{B 1} \cdot \mu_{P Z 3}+d \cdot s \cdot \lambda_{Z B 1} \cdot \lambda_{Z B 2}}{a \cdot b \cdot \lambda_{Z B 3} \cdot \mu_{P Z 3}+b \cdot \mu_{B 0} \cdot \mu_{B 1} \cdot \mu_{P Z 3}+\mu_{B 1} \cdot \mu_{P Z 1} \cdot \mu_{P Z 2} \cdot \mu_{P Z 3}-} \\
& -\lambda_{Z B 1} \cdot \mu_{P Z 1} \cdot \lambda_{Z B 3} \cdot \mu_{P Z 3}+a \cdot d \cdot s \cdot \lambda_{Z B 2} \cdot \mu_{P Z 2}+ \\
& +c \cdot d \cdot s \cdot \lambda_{Z B 1} \cdot \mu_{P Z 1}+d \cdot s \cdot \mu_{B 0} \cdot \lambda_{Z B 1} \cdot \lambda_{Z B 2}-a \cdot b \cdot c \cdot d \cdot s \\
& Q_{B}^{*}(s)=-\frac{b \cdot c \cdot \mu_{B 1}-\mu_{B 1} \cdot \lambda_{Z B 2} \cdot \mu_{P Z 2}+\lambda_{Z B 1} \cdot \lambda_{Z B 2} \cdot \lambda_{Z B 3}}{a \cdot b \cdot \lambda_{Z B 3} \cdot \mu_{P Z 3}+b \cdot \mu_{B 0} \cdot \mu_{B 1} \cdot \mu_{P Z 3}+\mu_{B 1} \cdot \mu_{P Z 1} \cdot \mu_{P Z 2} \cdot \mu_{P Z 3}-} \\
& -\lambda_{Z B 1} \cdot \mu_{P Z 1} \cdot \lambda_{Z B 3} \cdot \mu_{P Z 3}+a \cdot d \cdot s \cdot \lambda_{Z B 2} \cdot \mu_{P Z 2}+ \\
& +c \cdot d \cdot s \cdot \lambda_{Z B 1} \cdot \mu_{P Z 1}+d \cdot s \cdot \mu_{B 0} \cdot \lambda_{Z B 1} \cdot \lambda_{Z B 2}-a \cdot b \cdot c \cdot d \cdot s
\end{aligned}
$$

where: 


$$
\begin{gathered}
a=s+\lambda_{Z B 1}+\mu_{B 1} \\
b=s+\mu_{P Z 1}+\lambda_{Z B 2} \\
c=s+\mu_{P Z 2}+\lambda_{Z B 3}+\mu_{B 0} \\
d=s+\mu_{P Z 3}
\end{gathered}
$$

To obtain the relationships allowing to determine the probabilities of the electronic safety system staying in the considered states of: full fitness $\mathrm{S}_{\mathrm{PZ}}$, impendency over safety $\mathrm{S}_{\mathrm{ZB} 1}$ and $\mathrm{S}_{\mathrm{ZB} 2}$ and safety unreliability $\mathrm{S}_{\mathrm{B}}$, the further mathematical analysis should be conducted.

The presented considerations and the developed model allow for numerical assessment of various types of solutions (e.g. design and organisational ones) that can be implemented in order to minimise the impact of electromagnetic interference on the operation of the electronic safety systems.

In order to minimise the impact of electromagnetic interference on electronic safety systems, it is important to determine what is the source of interference, as well as what is a receiver and how the source is coupled with the receiver. There are three ways to reduce the propagation of interference:

- interference can be attenuated at the source (e.g. the use of protective shielding),

- manufacture of particular devices of the safety systems which will be insensitive to interference occurring in the electromagnetic environment (use of electronic elements created in the proper technology),

- minimising the transfer of interference by the coupling channel (e.g. decoupling filters, groundings, gate drives, shielding, etc.).

Therefore, if the developers know the electromagnetic environment conditions (i.a., through the above-presented measurements) in which the electronic safety system will be operated, then, the EMC requirements, which are usually known and can be considered in the device construction development, have to be met.

\section{Conclusion}

The proper functioning of electronic security systems operated at airports depends not only on the intensity of their use, but also on electromagnetic interference that affects them. Based on the observations, it can be concluded that artificially created or unintentional electromagnetic fields from different frequency ranges can have a negative effect on the functioning of electronic devices. Thus, there is a need to analyze the impact of electromagnetic interference on the 
operation of these systems. The article shows the measurements of the electromagnetic field generated by detectors which constitute a component of the electronic safety systems. Then, the author's operation process model including electromagnetic interference within low frequencies was also developed. The reliability-operational analysis of the electronic safety systems carried out by the authors allows for numerical assessment of various types of (technical and organisational) solutions, the objective of which is to minimise the impact of electromagnetic interference on the system operation. This is a new approach to this issue, as there are currently designed and installed security systems at airports, but there is no analysis of the impact of electromagnetic interference on their functioning. In further research concerning this issue, the authors plan to further develop reliability-operational models, which will differentiate between the states of partial fitness. It will allow for more accurate mapping of the operation of the electronic safety systems which are applicable at airports. Scientific considerations will be also taken to determine the security levels of alarm systems with taking into account the impact of strong electromagnetic pulses.

\section{References}

1. Burdzik R., Konieczny Ł., Figlus T.: Concept of on-board comfort vibration monitoring system for vehicles. In: Activities of Transport Telematics, TST 2013. Springer, Heidelberg 2013.

2. Charoy A.: Interference in electronic equipment. WNT, Warsaw 1999.

3. Dabrowski T., Bednarek M., Fokow K., Wisnios M.: The method of thresholdcomparative diagnosing insensitive on disturbances of diagnostic signals. Przeglad Elektrotechniczny - Electrical Review, Vol. 88, Issue: 11A, 2012.

4. Duer S., Zajkowski K., Płocha I., Duer R.: Training of an artificial neural network in the diagnostic system of a technical object. Neural Computing \& Applications 2013. Vol. 22, No. 7. DOI 10.1007/s00521-012-1052-9.

5. Kasprzyk Z., Rychlicki M.: Analysis of phiysical layer model of WLAN 802.11g data transmission protocol in wireless networks used by telematic systems. In: Proceedings of the Ninth International Conference Dependability and Complex Systems DepCoSRELCOMEX. Given as the monographic publishing series - „Advances in intelligent systems and computing”. Vol. 286. Springer, 2014.

6. Kierzkowski, A. \& Kisiel, T. Simulation model of security control system functioning: A case study of the Wroclaw Airport terminal. Journal of Air Transport Management. 2017. Vol. 64. Part B. P. 173-185. DOI 10.1016/j.jairtraman.2016.09.008. 
7. Kierzkowski A., Kisiel T.: Airport security screeners reliability analysis. In: Proceedings of the IEEE International Conference on Industrial Engineering and Engineering Management IEEM 2015. Singapore 2015.

8. Kovář S., Valouch J., Urbančoková H., Adámek M.: Electromagnetic interference of CCTV. In: International Conference on Information and Digital Technologies 2015. Zilina, Slovakia. DOI 10.1109/DT.2015.7222968.

9. Kováŕ S., Valouch J., Urbančoková H., Adámek M.: Impact of security cameras on electromagnetic environment in far and near-field. In: International Conference on Information and Digital Technologies (IDT) 2016. Rzeszow, Poland. DOI 10.1109/DT.2016.7557166.

10. Kozuba J., Sarnowski W.: Logistical process in military aviation organization, Scientific Journal of Silesian University of Technology. Series Transport 2017, Vol. 94. DOI 10.20858/sjsutst.2017.94.8

11. Lheurette E. (ed.): Metamaterials and Wave Control. ISTE and Wiley, 2013.

12. Łubkowski P., Laskowski D.: Selected issues of reliable identification of object in transport systems using video monitoring services. In: Communication in Computer and Information Science. Springer Berlin Heidelberg, Switzerland. Vol. 471, 2015, DOI 10.1007/978-3-662-45317-9_7.

13. Młyńczak J., Hejczyk T., Wszołek B., Gałuszka A., Surma D., Ogaza R., Burdzik R.: Passenger safety and information module in intelligent integrated traffic management system. Vibroengineering PROCEDIA Vol. 6, 2015.

14. NO-04-A004-9:2016. Obiekty wojskowe - Systemy alarmowe - Część 9: Wymagania dotyczące monitorowania alarmów. Warszawa: Wojskowe Centrum Normalizacji. [In Polish: Military installations - Alarm systems - Part 9: Requirements concerning monitoring of alarms. Warsaw: Military Centre for Standardization.]

15. Ott H.W.: Electromagnetic compatibility engineering. Wiley, 2009.

16. Paszek J., Kaniewski P.: Simulation of random errors of inertial sensors. In: 13th International Conference on Modern Problems of Radio Engineering, Telecommunications and Computer Science (TCSET). Lviv-Slavske, Ukraine, 2016.

17. PN-EN 50131-1:2009. Systemy alarmowe - Systemy sygnalizacji włamania i napadu - Część 1: Wymagania systemowe. Warszawa: Polski Komitet Normalizacyjny. [In Polish: Alarm systems - Intrusion and hold-up systems - Part 1: System requirements. Warsaw: Polish Committee of Standardization.]

18. Siergiejczyk M., Paś J., Rosiński A.: Issue of reliability-exploitation evaluation of electronic transport systems used in the railway environment with consideration of electromagnetic interference. IET Intelligent Transport Systems. 2016. 10 (9), DOI 10.1049/iet-its.2015.0183.

19. Skorupski J., Uchroński P.: Fuzzy Inference System for the Efficiency Assessment of Hold Baggage Security Control at the Airport. Safety Science Vol. 79, 2015. DOI 10.1016/j.ssci.2015.06.020. 
20. Skorupski J., Uchroński P.: Managing the Process of Passenger Security Control at an Airport Using the Fuzzy Inference System. Expert Systems With Applications, Vol. 54, 2016. DOI 10.1016/j.eswa.2015.11.014.

21. Stawowy M.: Model for information quality determination of teleinformation systems of transport. In: Proceedings of the European Safety and Reliability Conference ESREL 2014. CRC Press/Balkema, 2015.

22. Stawowy M., Kasprzyk Z.: Identifying and simulation of status of an ICT system using rough sets. In: Proceedings of the Tenth International Conference on Dependability and Complex Systems DepCoS-RELCOMEX. Given as the monographic publishing series - Advances in intelligent systems and computing. Vol. 365. Springer, 2015. DOI 10.1007/978-3-319-19216-1 45.

23. Urbančoková H., Valouch J., Adámek M.: Testing of an Intrusion and Hold-up Systems for Electromagnetic Susceptibility - EFT/B. International Journal Of Circuits, Systems And Signal Processing, Vol. 9, 2015.

24. Valouch J.: Technical requirements for Electromagnetic Compatibility of Alarm Systems. International Journal Of Circuits, Systems And Signal Processing, Vol. 9, 2015. 